scholars, a distinguished artist, and numbers of persons of more than average culture; yet there appears to be no correspondence between the mind and the length of the index finger.

R. A. N.

\section{Extraordinary Tides}

IN Iast week's "Notes" you say, speaking of the unprecedentedily high tide of the 15 th inst., that "no one seems to have expected an unusual tide." Allow me to state, sir, that in the Spectator of Nov. 7 th, 1874 , I predicted this extraordinary tide. As a matter of fact, the tide was higher than that of March 1874 , through the unusually swollen state of the river by floods and the N.W. gale.

No extraordinary tide can occur this side of March 1878 .

4, Buccleuch Place, Dulwich

B. G. JENKINS

\section{Further Linkage Work}

IN the interesting communication to NATURE, vol, xii. pp. 214-216, Prof. Sylvester gives account of the Hart and the Sylvester-Kempe "linkages." Of four points, three have work assigned. Problem: To employ the nondescript point.

First.-The Hart linkage yields the Cissoid, exactly as that curve is defined. Thus, with $p$ circling, $q$ resting, and $s$ tracing straight line ; $r$ traces cissoid. Second. - The Sylvester-Kempe linkage yields the Hyperbola, in that curve's simplest vector form. Thus, with $r$ opposite, to $p$ resting, and $p q, p s$ in constant directions ; $r$ traces the hyperbola whose assymptotes are these directions.

Burntisland, N.B.

\section{A Criminal Dog:}

I was so much pleased with the anecdote in Nature (vol. xiii. p. 36) of the criminal dog who buried the cat he had murdered, that I told my wife; but I did not mention the breed of the dog.

She said, "Was it not a retriever? becanse they always bury their food, so that it may become high." I could but answer that it was a retriever, and added that $I$ was afraid she had hit upon the real reason for the act.

General Post Office, Nov, I2

\section{OUR ASTRONOMTCAL COLUMN}

The Binary Star 44 Bootis.-In No. 2,064 of the Astronomische Nachrichten, Dr. Doberck, of Markree Observatory, has given an orbit of this binary, and a comparison with measures to the present year. The elements are--

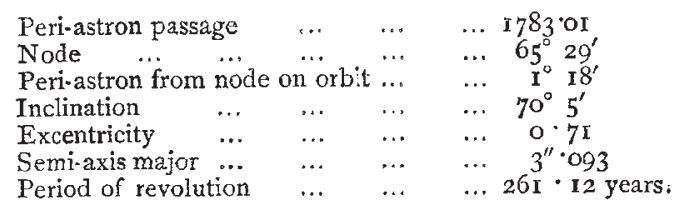

Dr. Doberck makes no reference to Sir W. Herschel's se ond measure in 1802 , giving for the angle $62^{\circ} 59^{\prime}$, or less than $3^{\circ}$ in advance of his measure I78 I, August I7. when the companion was first detected. The measure of I 802 was registered s.p. and "corrected by a subsequent observation to n.f.," as Sir John Herschel states in Memoirs R.A.S. vol.v. p. 46 ; but it is now pretty evident, from a projection of all the measures to 1875 , that the quadrant was correctly registered s.p., and consequently the angle for $1802 \cdot 246$ should be $207^{\circ} I^{\prime}$ according to our present method of reckoning. The angle calculated for this time from the above orbit is $206^{\circ} 55^{\prime}$, and this close agreement must be taken as very satisfactory evidence that Dr. Doberck has given us something like the true orbit, notwithstanding the difficulty of the case. It had been surmised that $\mathrm{Sir}$ W. Herschel's measures were to be increased $180^{\circ}$, Struve, in 1819 , obtaining an angle of $228^{\circ}$, and Herschel and South, in I821, $229^{\circ}$, but the position of the companion on the preceding side of the principal star, instead of the following side where it had been seen in $I 78 r$, was accounted for both by Sir John Herschel in his "Micrometrical Measures of 364 Double Stars," and by Struve in "Mensuræ Micrometricæ," by supposing motion in an orbit passing nearly through the eye of the observer, with the longer axis of the ellipse but slightly inclined to the meridian. Dr. Doberck finds an inclination to the tangent plane of the heavens, of $70^{\circ}$. Calculating from his elements, the following appear to have been the angles and distances from 1785 to 1800 :-

\begin{tabular}{|c|c|c|c|c|c|}
\hline $1785^{\circ} \mathrm{O}$ & $\ldots$ & Position & $74^{\circ} \circ$ & $\ldots$ & Distance \\
\hline $90^{\circ} 0$ & $\cdots$ & $"$ & $98 \cdot 3$ & $\cdots$ & $"$ \\
\hline 92.5 & $\ldots$ & " & 1320 & $\ldots$ & $"$ \\
\hline I $795^{\circ} \mathrm{O}$ & $\ldots$ & " & 1627 & $\ldots$ & $"$ \\
\hline $1800^{\circ}$ & $\ldots$ & " & $199^{\circ} 0$ & $\ldots$ & ", \\
\hline
\end{tabular}

The calculated distance for epoch of Sir W. Herschel's measure in $178 \mathrm{I}$ is $\mathrm{O}^{\prime \prime} \cdot 89$, and for that of $1802, \mathrm{I}^{\prime \prime} \cdot 05$; the observed distances being only by estimation in diameters of the companion, giving " $\frac{1}{2}$ or $\frac{3}{4}$ diameter" of smaller star in 1781 , and "barely $\frac{1}{2}$ diameter" in 1802, with power 460 in both years, are perhaps sufficiently well represented, though very admissible correction to one or two of the elements may diminish the distance in 1802 or increase that in $178 \mathrm{I}$.

For comparison with future measures we have from Dr. Doberck's orbit-

\begin{tabular}{|c|c|c|c|c|c|}
\hline $1876 \cdot 0$ & & Position & $241^{\circ} \cdot 45$ & $\ldots$ & Distance \\
\hline 78.0 & $\cdots$ & 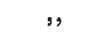 & $2417 x$ & $\cdots$ & . \\
\hline 80.0 & $\ldots$ & " & 24197 & $\ldots$ & ," \\
\hline
\end{tabular}

The Minor Planets.-In No. 35 of the Circulars of the Berliner Astronomisches Fahrbuch, Prof. Tietjen. notifies an arrangement which has been entered into by the Observatories of Leipsic, Leyden, Lund, Pola, and Vienna, for the more systematic and regular observation of the small planets. These Observatories have agreed to report to the editor of the 7 ahrbuch, every fortnight, the names or numbers of the planets which have been observed, with the dates of observation and the limits within which the planets whose positions are more uncertain have been sought. These communications will be so timed that they may arrive at Berlin on the Ist and 15 th of the month, and will be there arranged, printed, and circulated. An invitation is extended to those Observatories where the small planets are occasionally, though not regularly, observed, to join in the proposed scheme. In the same manner the state of calculation as regards the various members of this group will be made known. TheMilan Observatory has already engaged itself to calcula tion for No, I 5 I.

Mr. Daniel Kirkwood, of Bloomington, Indiana, writes with reference to the resemblance which exists between the elements of certain minor planets, instancing as the most striking case that of No. 54, Alexandra, and No. I4I, Lumen. An inadvertent application of the angle usually designated $\omega$ (or the distance of perihelion from node) in the wrong direction, renders the similarity between the orbits of these planets somewhat less striking than in Mr. Kirkwood's communication, but there is nevertheless considerable resemblance, as the following figures will indicate :-

\begin{tabular}{|c|c|c|c|c|}
\hline & & Alexandra. & & Lumex. \\
\hline Lion & ... & $294^{\circ} \times 6^{\prime}$ & & $341^{\circ} 3$ \\
\hline & $\cdots$ & $313^{\circ} 49^{\prime}$ & $\ldots$ & $318^{\circ} 5$ \\
\hline clination ... & $\ldots$ & $1 \mathbf{I}^{\circ} 47^{\prime}$ & $\ldots$ & $\mathrm{II}^{\circ} 33^{\prime}$ \\
\hline xcentricity... & $\ldots$ & 0.1987 & $\ldots$ & 0.2233 \\
\hline Mean distance & $\ldots$ & 27093 & $\ldots$ & 2.7095 \\
\hline Period & $\ldots$ & 1628.9 days. & $\ldots$ & 1629 \\
\hline
\end{tabular}

THE ZODIACAL LIGHT.-Those who are interested in the observation of this phenomenon will do well to be on the alert during dark evenings in the winter months. The most conspicuous exhibitions of the light in this country during the last few years have occurred in the month of January, the long standing recommendation to 